\section{Assessment of Error in Synoptic-Scale Diagnostics Derived from Wind Profiler and Radiosonde Network Data}

\author{
Gerald G. Mace and Thomas P. ACKerman \\ The Pennsylvania State University, University Park
}

(Manuscript received 6 June 1995, in final form 11 December 1995)

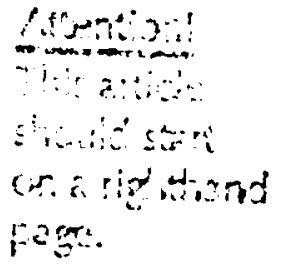

ABSTRACT

\section{Introduction}

The installation of the NOAA wind profiler demonstration network (WPDN) has significantly expanded opportunities for applying spatial objective analysis (SOA) techniques to data for diagnostic applications, particularly in the Kansas-Oklahoma region where a hexagonal network of profilers, spaced at approximately $175 \mathrm{~km}$ (Fig. 1), has been operational since mid 1991. These profilers operate at a frequency of $404.37 \mathrm{MHz}$ and provide horizontal wind observations in $250-\mathrm{m}$ vertical increments from $500 \mathrm{~m}$ above ground level to approximately $16 \mathrm{~km}$ with nominal 1$\mathrm{h}$ time resolution. The potential for WPDN data to characterize the meso-synoptic-scale state of the atmosphere has attracted several field programs to the midwestern United States. Among these are the second field phase of the First ISCCP (International Satellite Cloud Climatology Project) Regional Experiment (FIRE Cirrus II; NASA 1991) and the Atmospheric Radiation Measurement (ARM) Program (Stokes and Schwartz 1994). ARM has augmented the WPDN inner profilers with five radiosonde sites (Fig. 1) that collect soundings at high temporal resolution during frequent campaigns.

Integrating major field efforts as closely as possible with a dense spatial network of wind profilers seems an obvious choice. However, the scientific rationale be-

Corresponding author address: Dr. Gerald G. Mace, Department of Meteorology, The Pennsylvania State University, 503 Walker Building, University Park, PA 16802-5013. hind this choice deserves comment. In the case of FIRE Cirrus II, the choice was motivated by the possibility of diagnosing meso- and synoptic-scale vertical motions from divergence profiles deduced from profiler triangles. ARM seeks to extend this idea by attempting to diagnose the synoptic-scale advective tendencies of momentum and temperature from data for the purpose of integrating a single-column general circulation model (SCM). The SCM concept (Stokes and Schwartz 1994) relies on highly accurate diagnoses of the GCM-resolvable atmospheric state $(200-\mathrm{km}$ grid spacing and 1-h temporal resolution). The implied assumption is that the temporal history of five radiosonde profiles and seven wind profiles separated by approximately $200 \mathrm{~km}$ can accurately characterize the advective tendencies of the synoptic-scale atmosphere. In this paper we critically examine this assumption. In particular, we investigate the magnitude of objective analysis uncertainties and their effect on synoptic-scale diagnostic quantities valid at the center of the inner array of the WPDN.

A considerable amount of work has been published recently on spatial objective analysis of wind profiler and radiosonde data for the purpose of diagnostic analysis. Thiebaux and Pedder (1987) provide an excellent overall treatment of the subject, while recent articles by Davies-Jones (1993), Michaels (1994), and $\mathrm{Za-}$ mora et al. (1994) build on Thiebaux and Pedder's efforts. These works all consider the theoretical uncertainty of SOA techniques applied to data. The theoretical uncertainties, however, are typically quantified in terms of parameters not known a priori, that is, the scale length of dominant meteorological features or the 


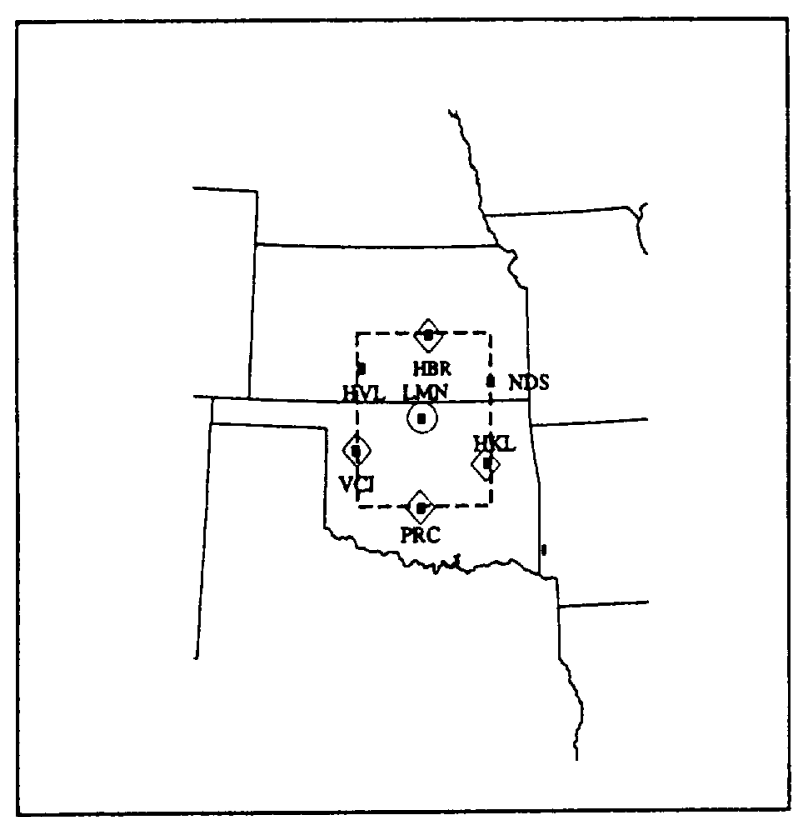

FIG. 1. Map of the inner array of the wind profiler demonstration array and the Atmospheric Radiation Measurement Program's (ARM) southern Great Plains (SGP) site. The filled squares mark the location of wind profilers. Diamonds show the location of special radiosonde launch facilities installed by ARM and the circle marks the location of the ARM central facility. The dashed rectangle shows the conceptual GCM grid box used by ARM in single-column modeling applications. Profiler locations are Haviland, Kansas (HVL), Hillsboro, Kansas (HBR), Neodesha, Kansas (NDS), Vici, Oklahoma (VCI), Purcell, Oklahoma (PRC), Haskell, Oklahoma (HKL), and Lamont, Oklahoma (LMN).

error in the observations. What is needed from the standpoint of the quasi-operational SCM approach envisioned by ARM is some practical quantification of objective analysis precision in terms of observable parameters, as well as a technique that will identify conditions where the uncerainties in the analysis products become large enough to negate further quantitative consideration. We address these issues in this paper. In the following section we briefly review SOA methodology and discuss theoretical limitations. To place the theoretical discussion into a more practical context, a case study is presented to illustrate the influence of objective analysis error on the diagnosis of horizontal divergence. In section three, we address the practical limitations of diagnosing field characteristics from small data networks using statistics derived from an observing system simulation experiment. Based on these statistics, a simple parameterization of objective analysis uncertainty is developed based on observable parameters.

\section{Methodology, uncertainty, and a practical} illustration

In order to place the following discussion in a rigorous context, it is helpful at this point to briefly review some of the applicable theoretical development found in Thiebaux and Pedder (1987) and Davies-Jones (1993). An observation $X(x, y, z, t)$ is assumed to have two basic components: $\mu(x, y, z, t)$ represents the portion of the observation containing the desired atmospheric signal and $\epsilon(x, y, z, t)$ accounts for the portion of the observation composed of observational error and atmospheric signal of unwanted scale. It is assumed further that $\epsilon$ is uncorrelated with $\mu$ in the spatial and temporal domains. The goal of the objective analysis technique is to minimize the influence of $\epsilon$ and to ensure the diagnosed characteristics are derived, as much as possible, from $\mu$. Following Thiebaux and Pedder (1987), a signal-to-noise ratio $\gamma$ is defined as the ratio of the mean-square deviations of the desired atmospheric signal to the mean square deviations of the noise,

$$
\gamma=\frac{\left\langle(\mu-\langle\mu\rangle)^{2}\right\rangle}{\left\langle(\epsilon-\langle\epsilon\rangle)^{2}\right\rangle}
$$

where the angle brackets denote the averaging operator applied to some number of spatially distributed observations. Owing to its assumed stochastic nature, $\langle\epsilon\rangle=0$ for some appropriate ensemble average. Therefore,

$$
\gamma=\frac{\left\langle(\mu-\langle\mu\rangle)^{2}\right\rangle}{\left\langle(\epsilon)^{2}\right\rangle}
$$

Since $\mu$ is never known a priori, maximizing $\gamma$ reduces to the problem of minimizing the sum of square residuals, $\left(g-X^{o}\right)^{2}$, where $g\left(x^{o}, y^{o}, z^{o}, t^{o}\right)$ is the analysis result at an observing location and $X^{\circ}$ represents the spatially distributed observations.

As shown by Thiebaux and Pedder (1987) and Davies-Jones (1993), all practical applications of spatial objective analysis, whether stated explicitly or not, make a determination as to the magnitude and importance of $\epsilon$ in a set of observations. For example, in the general technique described here, the field characteristics of the meteorological quantity are assumed to conform to some predetermined functional model. The characteristics of this model are imposed on the observations, leading to a spatially continuous mathematical approximation of the field. A straightforward example of this procedure is exact fitting of planar surfaces to data from station triangles. This includes Bellamy's (1949) graphical technique, line integral methods (Endlich and Clark 1963), and the linear vector point function (Zamora et al. 1987). Since the planar surface is fitted exactly to a minimal set of observations, no separation of signal from noise is possible. The implied assumption is that $\epsilon$ is of negligible consequence to the analysis result.

An immediate extension of the exact linear techniques is a generalized procedure that uses an arbitrary functional surface as the approximating model (in this work, we will consider polynomial surfaces). Thiebaux and Pedder show that this problem reduces to solving 


$$
\left[\left(\mathbf{F}^{o}\right)^{\mathrm{T}} \mathbf{F}^{o}\right] \hat{\boldsymbol{\theta}}=\left(\mathbf{F}^{o}\right)^{\mathrm{r}} \mathbf{X}^{o}
$$

for $\boldsymbol{\theta}$, provided that $\left[\left(\mathbf{F}^{\circ}\right)^{\mathrm{T}} \mathbf{F}^{o}\right]^{-1}$ exists. In Eq. (1), a spatially distributed set of observations is contained in the vector $X$, while the specified functional form of the assumed data field is contained in the so-called design matrix $\mathbf{F}$. The desired structural characteristics of the field variable (network mean and spatial derivatives) are returned in the vector $\theta$. When the number of observations exactly matches the number of variables required to define the functional surface, that surface is fitted exactly. However, when more observations are available than are needed to define the surface, the surface is fitted to the observations in a least squares sense by minimizing the sum of squared residuals between the analysis results and the observations. It can be shown easily that overdetermined least squares solutions effectively maximize the signal to noise ratio defined above.

The issue we seek to address is the degree of precision that can be ascribed to the diagnosed structural characteristics of the data field. Uncertainties in $\theta$ arise from observational noise, $\epsilon$ (contained in the vector $X$ ), and from the degree to which the actual field structure departs from the functional specification defined in the matrix $F$ (also known as truncation error). Assuming that errors in the observations follow their theoretical estimates, the former source of uncertainty can be estimated (Davies-Jones 1993), and this estimate should always be considered as a lower limit to the uncertainty in $\boldsymbol{\theta}$. The influence of $\epsilon$ in a set of observations tends to be minimized by spatially extensive and overdetermined data arrays. To evaluate the influence of truncation error, however, the actual spatial characteristics of the ambient field must be known. Since determining these spatial characteristics is typically the very purpose of the objective analysis exercise, the requisite knowledge needed to evaluate this error is generally unavailable, making truncation error an extremely troublesome source of uncertainty. Unlike observational error, truncation error tends to be minimized by decreasing the spatial extent of the data array used in Eq. (1), thus effectively sampling the feature under consideration in a more continuous fashion. For a fixed network of stations, this equates to decreasing the overdetermination of the functional surface and guarantees an increasing uncertainty in $\theta$ due to observational noise.

Consideration of the objective analysis uncertainty and its component sources must be predicated on a careful assessment of the overall analysis goals dictated by 1) the chosen scale of signal under investigation and 2) the degree of precision required in the diagnosed results. From a practical standpoint, it is generally impossible to separate uncertainty arising from observational noise and that due to truncation error in any given solution of Eq. (1) applied to data. With the exception of obviously erroneous observations, the observational noise component $c$ in a particular observation is both unknown and often not wholly uncorrelated from $\epsilon$ in observations from other nearby instruments as was assumed in the development of Eq. (1). Beyond this, the magnitude of truncation error can, at best, only be estimated crudely. The objective analysis exercise, then, becomes one of minimizing the resultant uncertainty arising from these oppositely trending sources of error and recognizing when the total uncertainty becomes so large, relative to the stated analysis goals, to negate further quantitative consideration of the results.

In our particular application (diagnosing synopticscale quantities for a single-column GCM), we adopt the ARM convention and consider the entire inner array of the WPDN as approximating a single-grid cell. We then examine planar polynomial approximations to the data fields that use between three and seven observations. The observing site configurations are approximately centered on the inner array centroid near Lamont, Oklahoma. For brevity we denote the particular objective analysis model by the number of observations used. Exact linear solutions using station triangles, for example, are termed L3, while an overdetermined linear solution to data from all seven inner array profilers is denoted by L7. The atmospheric signal we wish to resolve has dominant horizontal scales greater than $1000 \mathrm{~km}$ and we assume, following Holton (1979), that the quantities of interest scale as listed in Table 1. Establishing an accuracy standard for the diagnosed quantities is a somewhat ambiguous task that reduces to determining how much error is acceptable in any given realization of each quantity. Clearly the diagnosed quantities must be more accurate than the range over which that quantity scales. Reasoning that the inner array of the WPDN spans approximately one-quarter of a typical synoptic wave, we postulate that an objective analysis uncertainty less than $25 \%$ of the scale value implies a well-resolved quantity, while an uncertainty between $25 \%$ and $50 \%$ of the scale value implies a marginally resolved quantity. Uncertainties in excess of $50 \%$ of the scale value is considered poorly resolved.

A lower limit to the uncertainty in the diagnostic quantities can be estimated using the accepted ms uncertainty in the observations and the techniques described by Thiebaux and Pedder (1987) and Davies-

TABLE 1. Typical magnitudes of synoptic-scale quantities. Div and Vor denote horizontal divergence and relative vorticity. respectively.

\begin{tabular}{ll}
\hline$u_{v} v$ & $10 \mathrm{~m} \mathrm{~s}^{-1}$ \\
$T$ & $5 \mathrm{~K}$ \\
$\partial u / \partial x, \partial u / \partial y, \partial v / \partial x, \partial v / \partial y$ & $1 \times 10^{-5} \mathrm{~s}^{-1}$ \\
$\partial T / \partial x, \partial T / \partial y$ & $0.5 \times 10^{-5} \mathrm{~K} \mathrm{~m}^{-1}$ \\
$\operatorname{Div}, V_{0 r}$ & $1.4 \times 10^{-5} \mathrm{~s}^{-1}$ \\
$\left(V \cdot \nabla_{h} u\right),\left(v \cdot \nabla_{h} v\right)$ & $10 \times 10^{-5} \mathrm{~m} \mathrm{~s}^{-2}$ \\
$\left(V \cdot \nabla_{h} T\right)$ & $5 \times 10^{-5} \mathrm{~K} \mathrm{~s}^{-1}$ \\
\hline
\end{tabular}


TABLE 2. Uncertainty in objectively analyzed quantities at the center of the WPDN inner array due only to rms observational error. The observing sites used are: $L 3-H V L, N D S, P R C ; L A-V C l, P R C$, HKL, HBR; L5-HVL, HBR. NDS, PRC, VCl; L6-HVL, NDS, HBR. PRC, VCl, HKL (see Fig. 1 for profiler locations). An ms observational error of $1.5 \mathrm{~m} \mathrm{~s}^{-1}$ is assumed for the horizontal wind components and $0.5 \mathrm{~K}$ for the temperature. For the advective acceleration, the error corresponds to the individual components of the vector quantity.

\begin{tabular}{|c|c|c|c|c|}
\hline & L3 & $\mathrm{LA}$ & LS & L6 \\
\hline $\begin{array}{l}\text { u. v }\left(\mathrm{m} \mathrm{s}^{-1}\right) \\
T(\mathrm{~K})\end{array}$ & $\begin{array}{l}0.90 \\
0.25\end{array}$ & $\begin{array}{l}0.76 \\
0.26\end{array}$ & $\begin{array}{l}0.71 \\
0.24\end{array}$ & $\begin{array}{l}0.62 \\
0.21\end{array}$ \\
\hline$\frac{\partial(u, v)}{\partial x}\left(10^{-5} s^{-1}\right)$ & 0.69 & 0.68 & 0.59 & 0.48 \\
\hline$\frac{\partial(u, v)}{\partial y}\left(10^{-5} s^{-1}\right)$ & 0.65 & 0.54 & 0.51 & 0.48 \\
\hline$\frac{\partial T}{\partial x}\left(10^{-5} \mathrm{~K} \mathrm{~m}^{-1}\right)$ & 0.23 & 0.23 & 0.20 & 0.16 \\
\hline$\frac{\partial T}{\partial y}\left(10^{-5} \mathrm{~K} \mathrm{~m}^{-1}\right)$ & 0.21 & 0.17 & 0.17 & 0.16 \\
\hline $\begin{array}{l}\text { Div. Vor. }\left(10^{-5} \mathrm{~s}^{-1}\right) \\
\text { Adv. accel. }\left(10^{-5} \mathrm{~m} \mathrm{~s}^{-2}\right) \\
\text { Temp. adv. }\left(10^{-5} \mathrm{~K} \mathrm{~s}^{-1}\right)\end{array}$ & $\begin{array}{l}0.95 \\
7.8 \\
4.5\end{array}$ & $\begin{array}{l}0.87 \\
7.1 \\
4.2\end{array}$ & $\begin{array}{l}0.78 \\
6.4 \\
3.8\end{array}$ & $\begin{array}{l}0.68 \\
5.6 \\
3.3\end{array}$ \\
\hline
\end{tabular}

Jones (1993). Assuming the hourly horizontal wind components are known to within about $1.5 \mathrm{~m} \mathrm{~s}^{-1}$ (Strauch et al. 1987) and radiosonde-observed temperatures are known to within $0.5 \mathrm{~K}$ (Belt and Fuelberg 1982), the theoretical uncertainties in the diagnostic quantities of interest (those listed in Table 1) are shown in Table 2. Not surprisingly, the wind components and temperature are well resolved for all the objective analysis models considered. These quantities can be interpolated to the array centroid with an accuracy less than or equal to the assumed observational uncertainty. However, the first-order spatial derivatives and terms derived from them do not fare so well. In general, quantities derived from both station triangles and the fourstation network just marginally resolve synoptic-scale variability. While the situation improves somewhat for data networks of from five to seven observing sites, none of the quantities that rely on the first-order spatial derivatives are well resolved. Given that the level of precision listed in Table 2 is the maximum that can be expected from objectively analyzed data, any additional uncertainty brought about by larger than anticipated observational error or from any truncation error is indeed a serious concern.

To illustrate this latter point, we present a case study designed to highlight the influence of nonlinearities in the wind field and their influence on diagnosed horizontal divergence. We consider two profiler triangles, the first composed of the HVL, NDS, PRC profilers and the second composed of the VCI, HKL, HBR profilers. We denote the triangles as $T 1$ and $T 2$, respectively. For comparison purposes, we also examine results from a quadratic surface fit to data from a station array composed of the LMN, VCI, HVL, HBR, NDS, and PRC profilers (see Fig. 1). Note that each of the triangles is very nearly equilateral, while the triangles share no common vertices and have nearly identical centroids. This particular profiler geometry allows us to examine the influence of truncation error on the wind field diagnostics. As long as the wind field varies smoothly over the inner array, planar and higher-order polynomial surfaces fit to data will return identical wind field diagnostics within the bounds of observational uncertainty. As the wind field increases in complexity relative to the geographic region bounded by the inner array, the wind field diagnostics derived from the data will diverge.

Wind profiler data from 1500 UTC 25 November $1991(1500 / 25)$ to 0900 UTC 27 November 1991 $(0900 / 27)$ are examined. During the $43-\mathrm{h}$ period, all inner array profilers were operational and generated data of reasonable quality. In order to simplify interpretation and suppress observational error in the hourly winds, data were smoothed with a low-pass filter (Kaylor 1977) to remove oscillations with a period of $6 \mathrm{~h}$, and less and missing values were replaced using an interpolation scheme described by Akima (1978) and Akima (1984). Less than $10 \%$ of the data were missing, however. The evolution of the wind field during the period is shown by the time-height section of wind profiles from the LMN profiler (Fig. 2). The salient features of this period include the jet streak entrance region observed at Lamont early in the case study and the associated trough passage at $0000 / 26$. Winds in the lowest layers remained west to southwesterly throughout this period. After $0300 / 26$, winds became westerly and decreased in speed through much of the troposphere in response to a nearly stationary trough and

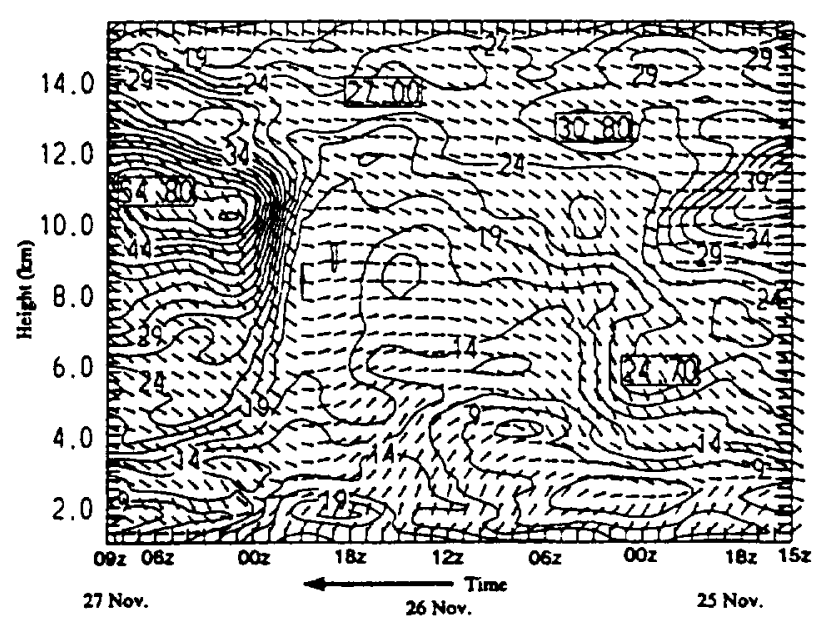

FIG. 2. Time-height cross section of horizontal winds observed by the Lamont. Oklahoma, profiler (site LMN in Fig. 1) from 1500 UTC 25 November 1991 to 0900 UTC 27 November 1991. Time runs from right to left in the diagram, contours are in meters per second, and vectors are compass direction (north being towards the top of the page) toward which the winds are blowing. 

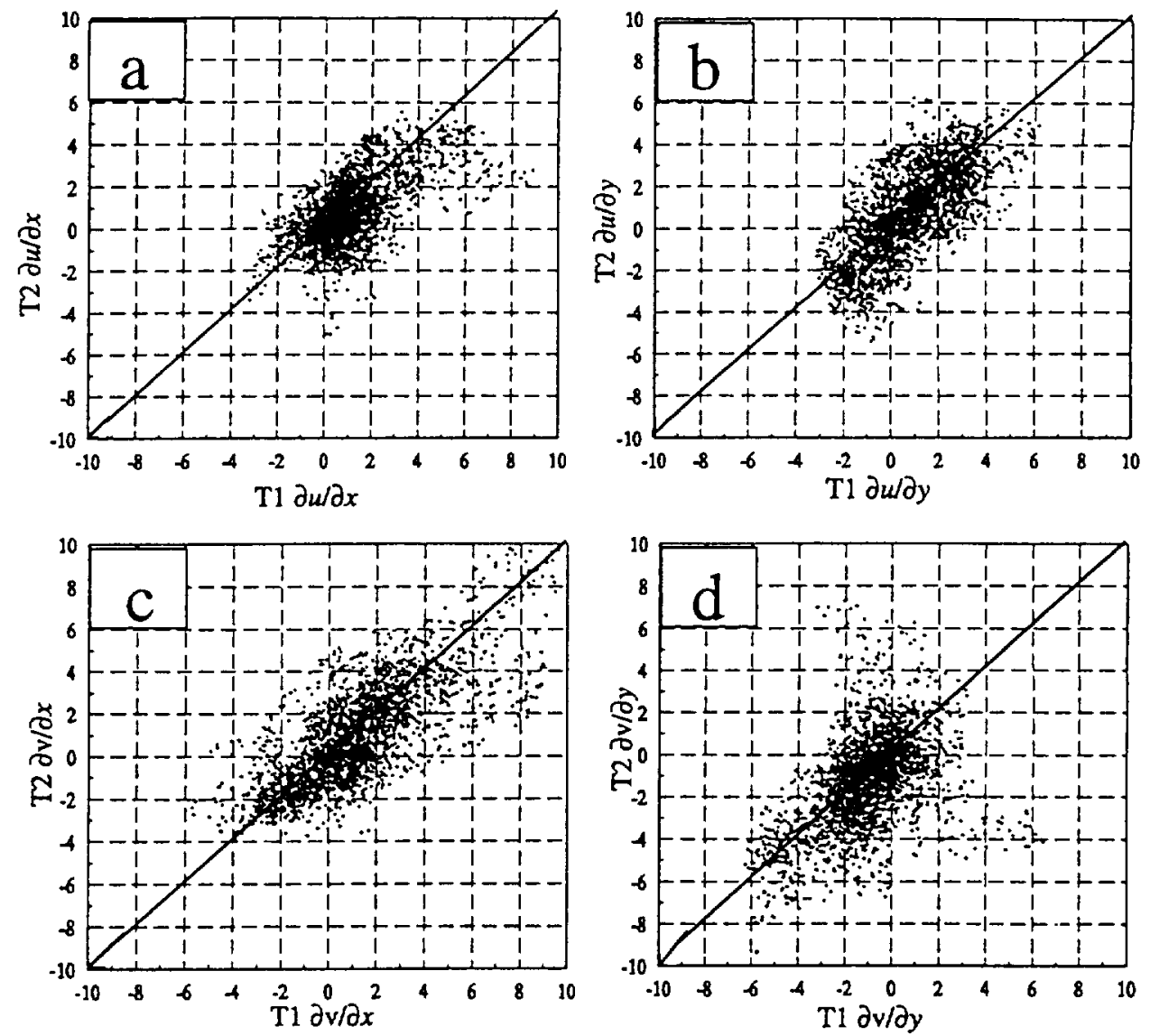

FIG. 3. Scatter plots of the horizontal velocity gradient components determined by exact linear fits (L3 objective analysis model) to viangles composed of the HVL, NDS, and PRC profilers (uriangle T1, abscissa) and the VCI, HKL, and HBR profilers (triangle T2, ordinate). Units are $10^{-5} \mathrm{~s}^{-1}$ : (a) $\partial u / \partial x$, (b) $\partial u / \partial y$, (c) $\partial v / \partial x$. (d) $\partial v / \partial y$.

diffluent flow pattern that was established over the central United States. After 1800/26, the winds backed further and an abrupt local acceleration occurred as the wind veered after $2300 / 26$

Figure 3 shows the correlation of the first-order spatial derivatives at all vertical levels estimated by the exact linear method applied to the two triangles. Some degree of linear correspondence exists in the scatter-

TABLE 3. The uncertainty in the indicated objectively analyzed quantities derived from the wind profiler polygons used in the case study. An rms observational error of $1.5 \mathrm{~m} \mathrm{~s}^{-1}$ is assumed and units are $10^{-5} \mathrm{~s}^{-1}$

\begin{tabular}{llcc}
\hline \hline & $\frac{\partial(u, v)}{\partial x}$ & $\frac{\partial(u, v)}{\partial y}$ & $\begin{array}{c}\text { Divergence, } \\
\text { Vorticity }\end{array}$ \\
\hline HVL, NDS, PRC & 0.69 & 0.65 & 0.95 \\
VCI, HKL, HBR & 0.87 & 0.74 & 1.1 \\
LMN, VCl, HVL, & 1.3 & 1.1 & 1.7 \\
HBR, NDS, PRC & 1.3 & &
\end{tabular}

plots and no obvious biases appear to exist in the data. However, significantly more scatter is evident in these plots than would be expected by considering only the influence of observational uncertainty on the analysis results (Table 3 ). Also, the scatterplot of $\partial v / \partial y$ suggests that a number of the diagnoses of this term display marked negative correlation.

Time series plots of the horizontal divergence and the component terms at two selected levels (Figs. 4 and 5) show that the degree to which the linear estimations differ is related to meteorological features that passed over the inner array. The $\partial u / \partial x$ term at $1.5 \mathrm{~km}$ shows close agreement to within the observational uncertainty through most of the period. The only significant disagreement between the linear estimates occurs near $0300 / 26$. A period of large uncertainty is also noted in the $\partial v / \partial y$ term between $0900 / 26$ and $1800 / 26$. Note that the horizontal divergence estimated using the quadratic fit at $1.5 \mathrm{~km}$ closely agrees with the linear estimation from triangle $\mathrm{T} 1$. This agreement is, however, fortuitous and due to a cancellation of differences 


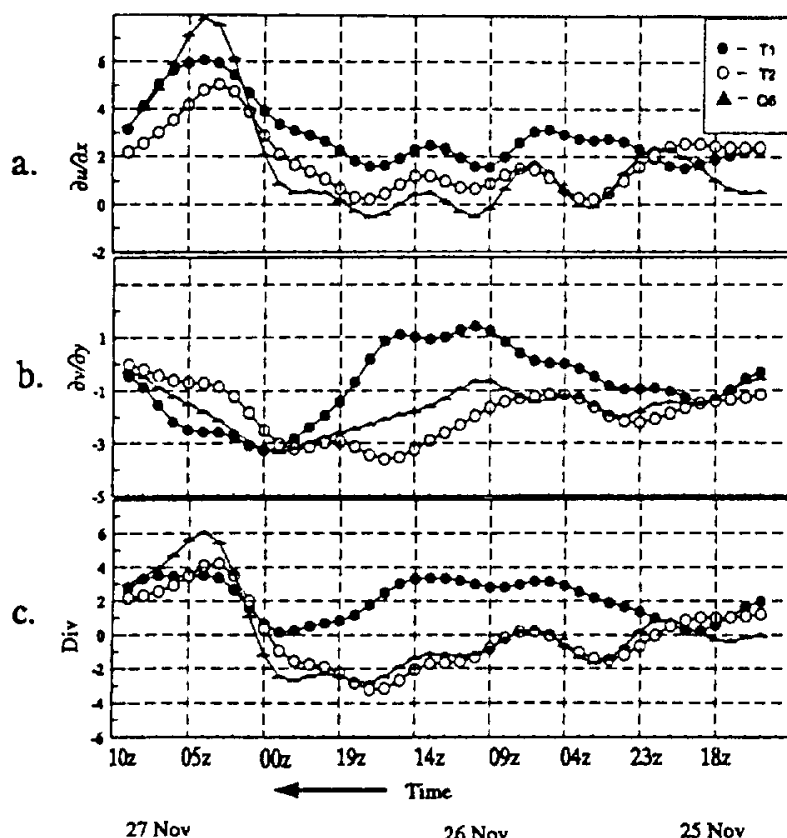

Frg. 4. Time series of (a) $\partial u / \partial x$, (b) $\partial u / \partial y$, and (c) horizontal divergence $(\partial w \partial x+\partial v / \partial y)$ calculated at $1.5 \mathrm{~km}$ using exact linear polynomial fits ( $\mathrm{L} 3$ objective analysis model) to wind profiler triangles composed of the HVL, NDS, and PRC profilers (triangle $\mathrm{Tl}$, solid circle) and the VCI, HKL, and HBR profilers (triangle T2, open circle). The curve denoted by solid triangles uses an exact quadratic fit (Q6 in the legend) to profiler data from the HVL. HBR. NDS, PRC. VCI, and LMN profilers. The abscissa has units of $10^{-3} \mathrm{~s}^{-1}$. Time runs from right to left as in Fig. 2 from 1400 UTC 25 November 1991 to 1000 UTC 27 November 1991.

in the individual terms as can be noted by examining Figs. $4 \mathrm{a}$ and $4 \mathrm{~b}$. A more accurate depiction of the uncertainty in the horizontal divergence is seen by comparing the two linear estimates where an average difference of $6 \times 10^{-5} \mathrm{~s}^{-1}$ occurs during this period. The magnitude of this difference is alarming since it is nearly as large as the expected range of atmospheric signal over an entire synoptic-scale system and more than an order of magnitude larger than the minimum uncertainty estimated using only the assumed $\mathrm{rms}$ error in the observations.

Large differences in the two linear estimates in the upper troposphere (Fig. 5) are evident throughout the case study. Agreement to within the observational uncertainty occurs in only 8 of the 43 hours under consideration. The linear solutions tend be negatively correlated much of the time and the quadratic solution tends to remain midway between them. This is most vividly displayed by the $\partial v / \partial y$ terms between $1800 / 26$ and $0400 / 27$. During this time a trough and jet streak exit region influenced the Oklahoma and Kansas region (Mace et al. 1995). It should be noted that this exit region was part of a synoptic-scale feature that passed rapidly over the middle United States and generated a propagating band of mid- and upper-level cloudiness.
In spatial objective analysis, the values of the diagnosed horizontal derivatives usually are considered valid at the observational centroids of the polygons. In this case, the centroids of the two triangles and the sixstation array are nearly identical, but the wind field characteristics derived from them are markedly different. This is a vivid example of the difficulties that must be considered when objective analysis results are to be used for quantitative applications such as calculation of vertical motions or construction of boundary conditions for single-column models. It is these types of strongly forced situations that must be accurately resolved if data-derived diagnostics are to be used successfully. For instance, the SCM concept adopted by ARM assumes that a meteorologically diverse and statistically significant set of well-characterized cases will compose the database against which cloud and radiation parameterizations will be tested (Stokes and Schwartz 1994). Periods contaminated by objective analysis error must be filtered from this database. Given the marginal precision of inner-array data polygons to resolve synopticscale signal, identification of poorly resolved cases is crucial if objectively analyzed wind profiler and radiosonde network data are going to be credible sources of information.

As demonstrated by the case study, the uncertainty in derivative estimates can easily be an order of magnitude larger than the uncertainty calculated by considering only the assumed uncertainty in the observations. This additional uncertainty arises due to a combination of truncation error and a spatial and temporal depen-

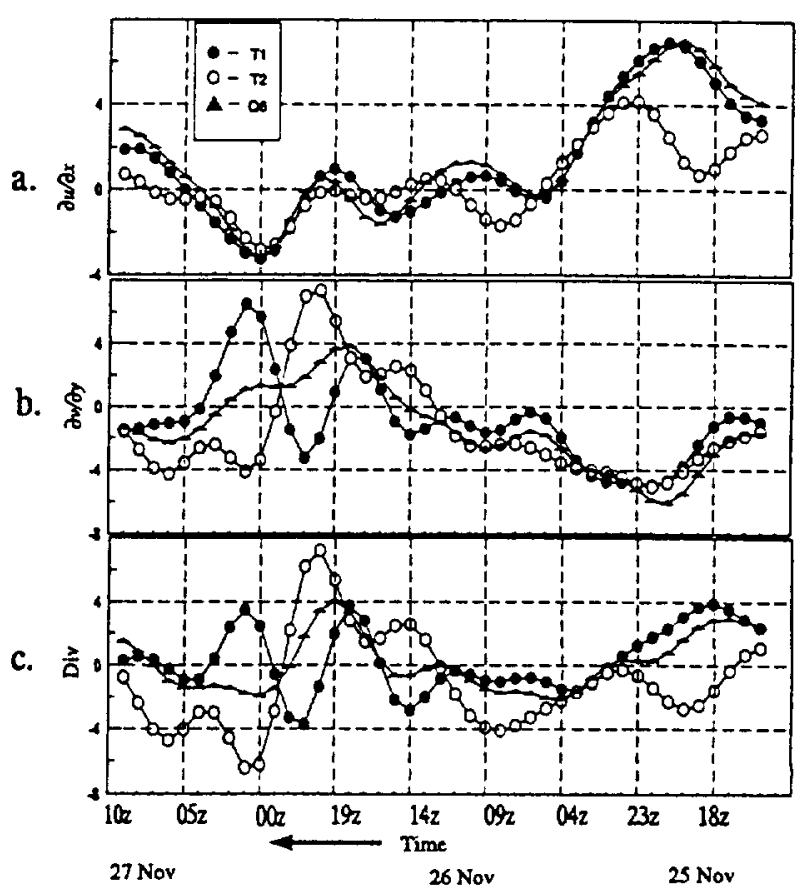

FIG. 5. As in Fig. 4 except at $9.5 \mathrm{~km}$. 
dence of observational error not accounted for in the development of Eq. (1). The point we wish to stress is that neither of these additional sources of uncertainty can be known a priori nor can they be evaluated rigorously in a postanalysis mode. Furthermore, it is generally impossible to evaluate separately the sources of objective analysis error in any given application of Eq. (1) to data. While the work of previous authors has been necessary to highlight theoretical limitations ( $\mathrm{Da}$ vies-Jones 1993; Zamora et al. 1994; Michaels 1994; Doswell and Caracena 1987), what is needed from a practical standpoint is some quantification of the precision that can be expected from applying objective analysis algorithms to data when a realistic convolution of both observational error and muncation error are possible. A straightforward technique is required that will aid the analyst in identifying situations where the uncertainty in derivative estimation is likely to be so large as to negate further quantitative consideration of a particular meteorological case.

\section{Estimates of precision and a technique for error discrimination}

In order to examine the precision of diagnostic quantities derived from data using the algorithms described in the previous section, some objective measure of the true atmospheric state is required. We have opted to use output from a mesoscale model as a surrogate for the true atmospheric state in an observing system simulation experiment (OSSE). Using a mesoscale model in this way has advantages over the analytical functions used by previous authors to examine objective analysis precision since a full spectrum of realistic meteorological conditions can be considered while the error characteristics of the observing systems can be simulated. By combining realistic atmospheric conditions with simulated observations, objective analysis errors can be quantified and the statistical precision of the objective analysis algorithms can be ascertained.

\section{a. OSSE Methodology}

Output from the Mesoscale Analysis and Prediction System (MAPS; Benjamin 1990) is used. The model output is produced on a $60-\mathrm{km}$ polar stereographic grid in pressure coordinates. Although wind profiler diagnostics are normally performed in geometric height coordinates, the vertical coordinate is largely irrelevant for the purpose of the experiment performed here since intercomparisons between quantities diagnosed from simulated observations and the model output are considered. Our goal is to create a statistical sample of objective analysis errors representative of the full continuum of meteorological situations that occur in the Kansas-Oklahoma region. Nearly the entire MAPS model domain (from approximately $20^{\circ} \mathrm{N}, 120^{\circ} \mathrm{W}$ in the southwest to $55^{\circ} \mathrm{N}, 60^{\circ} \mathrm{W}$ in the northeast) is, there-
TABLE 4. The data polygons used in the observing system simulation experiment (OSSE). See Fig. I for the geographic distribution of the sites. In the OSSE, the L.3 or LA polygon used in each case was determined randomly.

\begin{tabular}{ll}
\hline \hline L3 & HBR, VCl, HKL, PRC, HVL, NDS \\
L4 & HBR, VCl, HKL, PRC, HVL, HBR, NDS, PRC \\
L5 & HVL, HBR, NDS, PRC, VCl \\
L6 & HVL, HBR, NDS, HKL, PRC, VCl \\
\hline
\end{tabular}

fore, considered and the period examined extends over many months.

Starting at the northwest corner of the model grid and the lowest vertical level, an array of simulated observing points is defined, identical in their spatial distribution to the inner array of the WPDN. The data at the MAPS grid points within the region bounded by the array of simulated observing sites are used to ascertain the actual area-averaged diagnostic terms. The areaaveraged quantities derived from the model gridpoints include the horizontal wind components, temperature, wind component and temperature gradient terms, the horizontal velocity divergence, relative vorticity, temperature advection, and advective acceleration. These area-averaged quantities calculated from the model grid points are taken to represent $\mu$ or the desired part of the synoptic-scale atmospheric signal.

Simulated observations of the wind components and temperature are determined at the prescribed observing sites from the model gridpoint data. Temperature profiles are generated by interpolating the model-predicted temperature to the observing site locations. Unlike radiosonde data, however, wind profiler hourly data do not necessarily represent single-time observations but are composed generally of temporal means calculated from filtered 6-min Doppler moments. Therefore, in order to simulate faithfully actual wind profiler observations, the model's wind field is used to reconstruct approximately what a profiler would observe at a particular vertical level. We construct a wind field streamline that represents 30 -min upstream and 30-min downstream advecting distances. The horizontal wind components are sampled at ten points equally spaced in time along the streamline. The wind velocity at these points is taken to represent the eastward and northward moments data. The components are used to create the simulated wind profiler hourly values. This technique assumes no vertical advection and stationarity in the dynamical evolution of the wind field. While this procedure does not reproduce actual wind profiler observations exactly, it is more realistic than simply taking the gridpoint wind values at the observing locations. The largest differences between the gridpoint data and the simulated profiler data tend to be on the order of 4 $\mathrm{m} \mathrm{s}^{-1}$ and occur near jet entrance and exit regions. The mean difference tends to be less than $1 \mathrm{~m} \mathrm{~s}^{-1}$.

Following the example of Belt and Fuelberg (1982), random error is added to each of the simulated obser- 

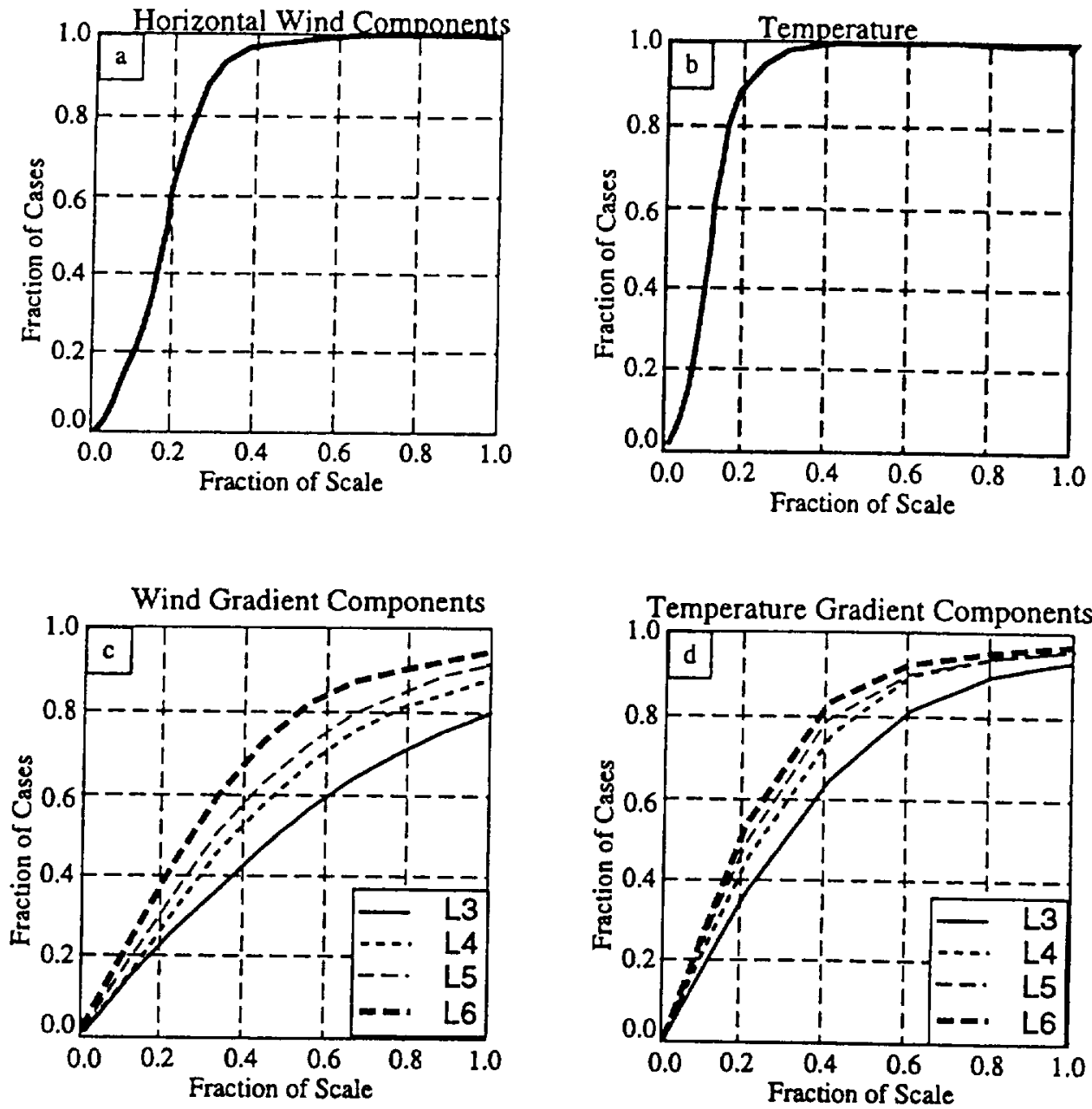

FiG. 6. Cumulative error statistics from the OSSE for (a) wind components. (b) temperature, (c)-(h) the indicated derivative-related quantities, with a key showing which objective analysis model is used in the corner of each graph. Abscissa values are fractions of the scale magnitudes listed in Table 1.

vations composing the seven vertical profiles. The observational error added to the observations has an ms value of $1.5 \mathrm{~m} \mathrm{~s}^{-1}$ for the wind components and $0.5 \mathrm{~K}$ for the temperature. The perturbed data are then used as input to the objective analysis algorithms [Eq. (1)] where planar surfaces are fitted to various polygons of observing sites ranging from triangles to the full hexagonal network. The polygons used in this exercise are listed in Table 4. The objective analysis results and the simulated data are differenced from the model's areamean quantities and the differences stored as histogram counts that are a function of rms observational error and diagnostic quantity.

This procedure is followed at each model level. After the full vertical column has been considered at a particular location, the simulated observational array is moved eastward three grid points and the process is repeated. After the entire model domain has been examined, the next MAPS analysis (typically $3 \mathrm{~h}$ later) is processed similarly. We have examined model output from late March 1994 to late October 1994, resulting in approximately $10^{8}$ separate comparisons for each polygon type. While this OSSE is able to simulate many aspects of the observational data, certain characteristics of the data are not accounted for. These include the influence of small-scale phenomena on the wind and temperature observations. Also, we did not attempt to simulate the advection of radiosondes with the wind, nor was any attempt made to simulate situations where the observational noise was correlated across the data network. The results presented below are, therefore, slightly biased toward lower error.

\section{b. OSSE results}

The results of the OSSE are presented in terms of cumulative percentile statistics in Fig. 6 . The horizontal wind components and temperature (Figs. $6 \mathrm{a}$ and 6b) 

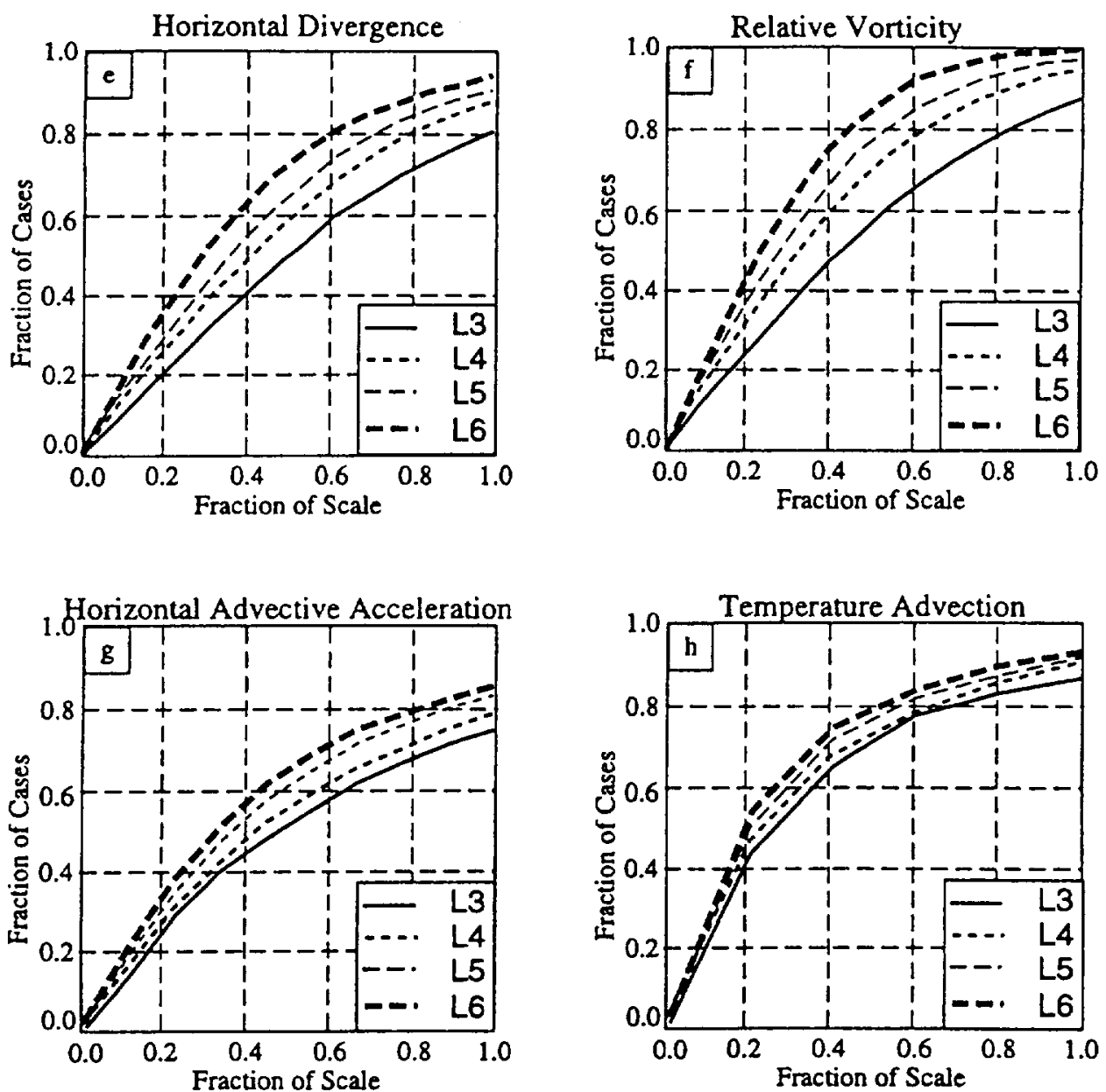

FIG. 6. (Continued)

demonstrate no sensitivity to the objective analysis model used and can generally be estimated with high precision (relative to their scales). The wind components are well resolved (within $25 \%$ of their scale value) more than $80 \%$ of the time and are marginally resolved (within $50 \%$ of their scale) $98 \%$ of the time. Objectively analyzed temperature estimates demonstrate a somewhat higher degree of precision, being well resolved in $96 \%$ of cases and marginally resolved 99\% of the time. Experiments were also performed with higher levels of rms error in the temperature observations (not shown). The motivation for this is the installation of temperature profiling remote sensors known as radio acoustic sounding systems (RASS) at several profiler sites. These instruments provide continuous soundings of virtual temperature through the lower troposphere but with somewhat less precision than radiosonde observations. For $\mathrm{ms}$ observational uncertainty more typical of RASS $(1.5 \mathrm{~K})$, less than $10 \%$ of cases were well resolved and less than $50 \%$ of cases were interpolated with marginal precision.
The sensitivity to the objective analysis model becomes more obvious when considering the spatial derivative terms and quantities derived from them. As expected, the degree of precision that can be anticipated depends in a straightforward manner on the degree of overdetermination of the objective analysis model. Exact planar fits using data triangles tend to demonstrate the minimum precision, while the planar objective analysis model utilizing six data points as input tends to be most accurate. In general, regardless of the number of data points used in the objective analysis model, the majority of all cases can be estimated to within a particular quantity's scale value. In other words, the correct sign of a particular term can be estimated accurately in the majority of cases. For quantitative application, however, the fraction of cases demonstrating a high degree of precision (within 25\% of the scale value) is significantly less than found for the wind components and temperature. Consider, for instance, the wind gradient components. The exact planar model demonstrated precision to within $25 \%$ of the scale 
value only $31 \%$ of the time. The fraction increases steadily as data points are added to the planar objective analysis model with the L6 model being well resolved just over one-half of the time.

Similar conclusions are evident for the remaining terms and are summarized in Table 5 . In general, the temperature gradient terms and the horizontal advective tendency of temperature tend to be resolved with high precision about one-half of the time. These statistics improve somewhat for the L5 and L6 models. Divergence and vorticity can be estimated with good accuracy about one-third of the time when using triangles of observing sites, and, as before, the degree of overdetermination in the planar surface tends to increase the fraction of cases. The improvement is most clearly demonstrated in relative vorticity, with the fraction improving to $57 \%$ for the L6 model, while the divergence is well resolved less than one-half the time, regardless of the model considered. The statistics for the advective acceleration are similar, except that even less improvement is noted in this quantity for the overdetermined linear models. The temperature gradient components and the associated temperature advection demonstrate somewhat higher precision, being well resolved in about $45 \%$ of cases for triangles and $63 \%$ and $55 \%$, respectively, for arrays of six temperature observations.

Objective analysis uncertainty must be considered a serious limitation to quantitatively diagnosing field characteristics from data using existing observational networks. While the spatial objective analysis techniques considered here are generally capable of determining the correct sign of a given quantity, moving beyond qualitative applications with any certainty is possible in only a surprisingly small fraction of cases. The low level of precision demonstrated in the terms derived from the spatial derivatives can be understood by considering the objective analysis precision statistics for the horizontal wind components and temperature. Even though the level of certainty in the wind components and temperature is high relative to their scales, the fraction of cases demonstrating precision to within $5 \%$ of $10 \%$ of the scale values are small. This indicates that small variations in the wind and temperature fields that occur naturally are not well characterized in the data. In essence, subtle objective analysis errors often dominate the actual physical variability of these fields, resulting in imprecise estimates of the horizontal gradients.

\section{c. Parameterization of the uncertainty}

The results presented above clearly indicate that the wind profiler and radiosonde data streams must be examined in close detail in order to identify those situations that are amenable to quantitative analysis. Manual inspection of the data, however, is not a viable option. Not only is a manual filter expensive in terms of time, but in many cases even a highly trained meteorologist
TABLE 5. The fraction of cases in which the diagnostic quantity calculated by the indicated objective analysis model had enror magnitude equal to or less than one-fourth of the scale value (top number) and equal to or less than one-half the scalc value (bottom number). The quantities listed are the horizontal uind components $(u, v)$, temperature $(T)$, wind gradient components (ug), temperature gradient components ( $(g)$, horizontal divergence (div), relative vorticity (vor), horizontal advective acceleration components (ua), and horizontal temperature advection (ta).

\begin{tabular}{ccccccccc}
\hline Model & u/v & $T$ & ug & tg & div & vor & ua & ta \\
\hline \multirow{2}{*}{ L3 } & 0.82 & 0.98 & 0.31 & 0.44 & 0.34 & 0.33 & 0.32 & 0.45 \\
& 0.99 & 0.99 & 0.50 & 0.71 & 0.50 & 0.51 & 0.50 & 0.68 \\
L4 & 0.84 & 0.99 & 0.40 & 0.53 & 0.38 & 0.42 & 0.36 & 0.49 \\
& 0.99 & 0.99 & 0.62 & 0.82 & 0.60 & 0.64 & 0.57 & 0.72 \\
L5 & 0.83 & 0.98 & 0.44 & 0.58 & 0.46 & 0.48 & 0.39 & 0.52 \\
& 0.99 & 0.99 & 0.68 & 0.84 & 0.64 & 0.78 & 0.62 & 0.75 \\
L6 & 0.86 & 0.99 & 0.52 & 0.63 & 0.46 & 0.57 & 0.42 & 0.55 \\
& 0.99 & 0.99 & 0.78 & 0.88 & 0.72 & 0.83 & 0.64 & 0.79 \\
\hline
\end{tabular}

would be unable to discriminate accurately between the subtle variability imposed by synoptic-scale signal and that imposed by objective analysis error. Situations such as that documented in the case study, as well as error caused by highly erroneous observations, are relatively easy to identify. More typically, however, the bias in the analysis results tends to occur over deep atmospheric layers and often appears wholly reasonable relative to the meteorology of the situation.

To remove the subjectivity and expense of manual filtering, we have attempted to parameterize the objective analysis uncertainty. When an objective analysis model is applied to an array of observations, estimates of the area-mean wind components and temperature $\left(u_{\text {est }}, v_{\text {esl }}\right.$, or $\left.T_{\text {est }}\right)$ are returned, as well as estimates of the first-order spatial derivatives. Assuming that observations of the wind components and temperature ( $u_{\mathrm{oos}}$. $\left.v_{\text {obs }}, T_{\text {obs }}\right)$ are available near the observational centroid (as at the ARM site near Lamont, Oklahoma), we attempt to parameterize the uncertainty in the objectively analyzed spatial derivatives in terms of the observed difference between the objectively analyzed and observed wind components and temperature. Hereafter we refer to this error in the objectively analyzed wind components and temperature as $\delta_{0}$ (i.e., $\delta_{0}=u_{\mathrm{est}}-u_{\mathrm{obs}}$ ) and the uncertainty in the objectively analyzed spatial derivatives as $\epsilon_{1}$.

The operating hypothesis of this parameterization is that a linear relationship exists between the cumulative statistics of $\delta_{0}$ and the cumulative statistics of $\epsilon_{1}$. This does not necessarily imply a direct relationship between $\epsilon_{1}$ and $\delta_{0}$ on a case by case basis but does imply that the fraction of cases exhibiting error of $\delta_{0}$ is, based on that fraction of cases, indicative of an upper limit for $\epsilon_{1}, \epsilon_{1}^{\max }$. This is illustrated schematically in Fig. 7 . Consider a particular situation where the difference between an observed horizontal wind component and the 


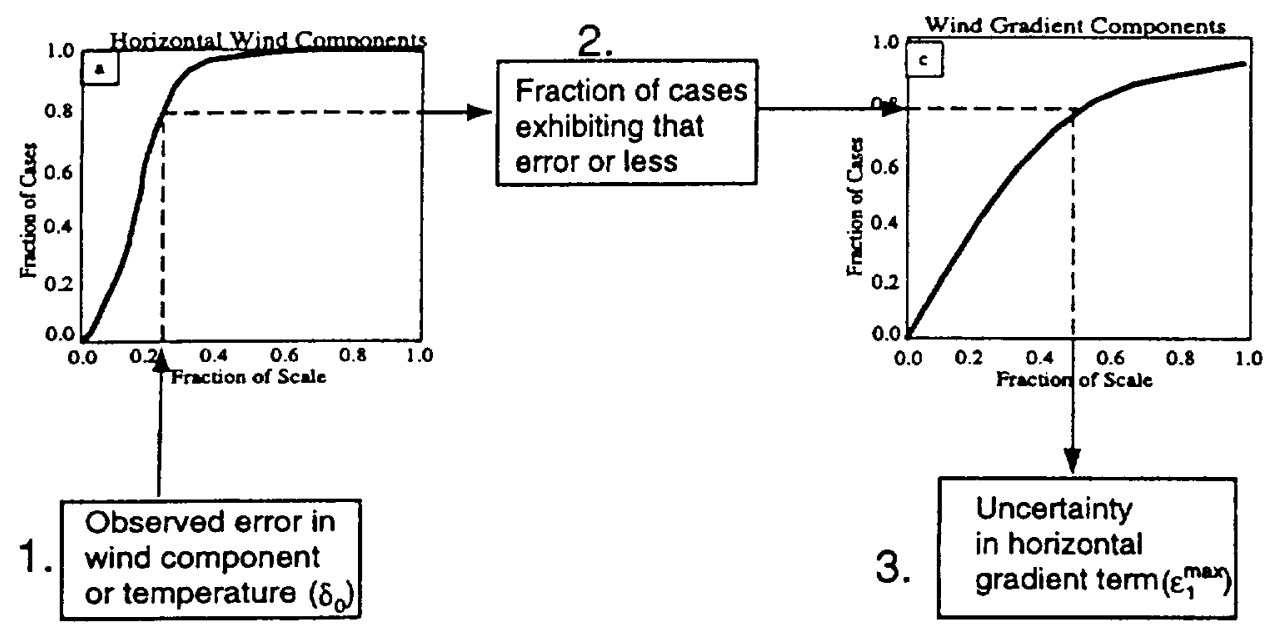

FiG. 7. Schematic illustration of the parameterization of maximum likely $\epsilon_{1}$ in terms of observed $\delta_{0}$. An upper limit to $\epsilon_{1}$ is estimated in terms of the observed value of $\delta_{0}$.

objectively analyzed horizontal wind components is 2.2 $\mathrm{m} \mathrm{s}^{-1}$. We find from the OSSE cumulative statistics that the objectively analyzed wind components are in error by $2.2 \mathrm{~m} \mathrm{~s}^{-1}$ or less $78 \%$ of the time. We assume that this cumulative fraction (78\%) is applicable to the other objective analysis products derived from the particular realization. In other words, the spatial gradient terms returned from the objective analysis model are as accurate as the most accurate $78 \%$ of cases as determined from the OSSE statistics. This equates to an uncertainty $\left(\epsilon_{1}^{\max }\right)$ in the horizontal spatial derivatives of one-half of the scale value or $0.5 \times 10^{-5} \mathrm{~s}^{-1}$.

To verify the parameterization of $\epsilon_{1}^{\max }$, we performed an identical OSSE to that described above except that $\epsilon_{1}$ was considered in terms of $\delta_{0}$ assuming an rms error of $1.5 \mathrm{~m} \mathrm{~s}^{-1}$ in the wind components and $0.5 \mathrm{~K}$ in the temperature. Results are presented in Figs. 8 and 9. We find that beyond some minimum $\delta_{0}$, the errors in the first-order terms are directly related to the error in the associated wind components and temperature, although this relationship is more evident for the horizontal wind than for the temperature. The relationship also tends to be strongest for the L3 model and tends to decrease as the degree of overdetermination in the objective analysis model increases.

The parameterization of $\epsilon_{1}^{\max }$ described above and shown schematically in Fig. 7 tends to overpredict the uncertainty in the spatial derivatives for $\delta_{0}$ greater than some critical value and underpredict the uncertainty for $\delta_{0}$ less than this critical value. We assumed that as $\delta_{0}$ approaches zero, $\epsilon_{1}^{\max }$ also approaches zero. This assumption fails for two reasons. First, owing to truncation error, there is some probability that for an incorrectly diagnosed spatial derivative, a correct estimate of the wind component or temperature will be returned. This effect is compounded by rms error in the obser- vations. These random errors tend to cancel for determination of the zeroth-order terms since the zeroth-order terms are approximately weighted averages of the observations. The spatial derivatives, however, are influenced by each observation and the error in each observation influences the diagnosed spatial derivatives. These factors limit our ability to parameterize $\epsilon_{1}^{\max }$ when $\delta_{0}$ decreases below some critical value $\left(\delta_{0}^{\lim }\right)$. This limit can be seen in Figs. 8 and 9 where the $\epsilon_{1}$ percentile curves decrease to a minimum value and remain constant $\left(\epsilon_{1}^{\mathrm{lim}}\right)$. The $\epsilon_{1}^{\mathrm{lim}}$ is essentially the maximum precision that can be prescribed to a diagnosis of the spatial derivatives. While $\delta_{0}^{\mathrm{Lim}}$ does not change appreciably for increasingly overdetermined planar models, $\epsilon_{1}^{\text {lim }}$ does tend to decrease as the number of observations is increased. Not only are the overdetermined linear models more accurate generally, but our ability to constrain the error bars on the resulting analysis product is enhanced. It should be noted, however, that $\epsilon_{1}^{\lim }$ tends to remain a significant fraction of the scale values of the quantities being considered. In other words, it is difficult to identify unambiguously those cases where the objective analysis results are more accurate than $\epsilon_{1}^{\lim }$. Not only do highly accurate characterizations of the spatial derivatives seldom occur, but it is difficult to know when they do occur.

Considering these results, a practical parameterization of $\epsilon_{1}^{\max }$ can be formulated. As before, we parame. terize $\epsilon_{1}^{\max }$ in terms of $\delta_{0}$ except that we follow the 90th percentile curves shown in Figs. 8 and 9. The equations for this parameterization are

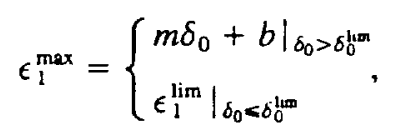


L3 Objective Analysis Model

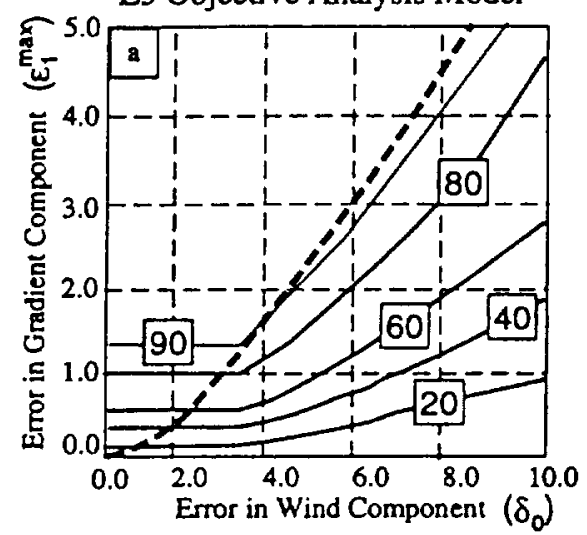

L5 Objective Analysis Model

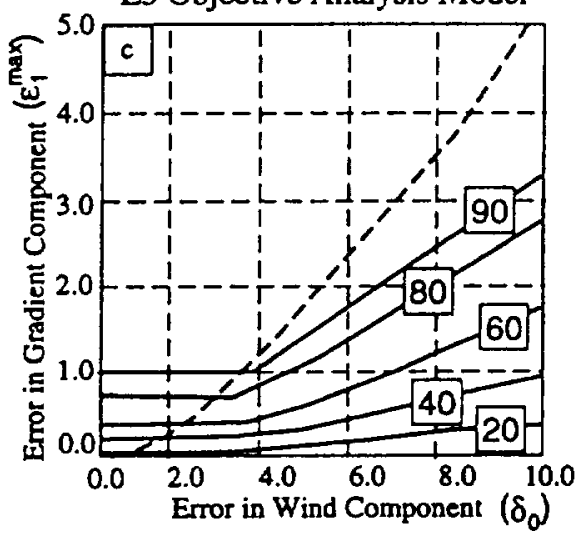

L4 Objective Analysis Model

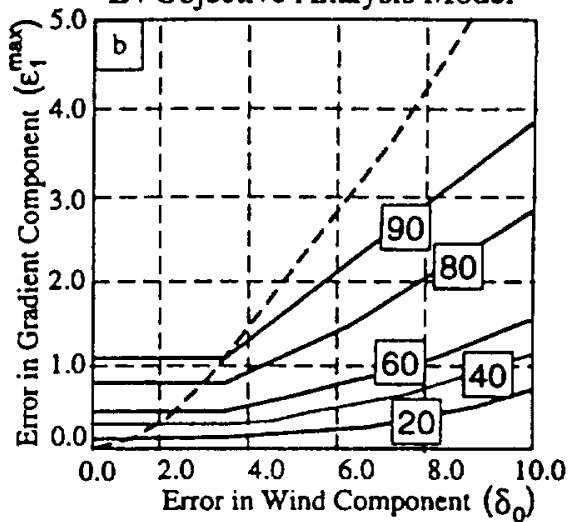

L6 Objective Analysis Model

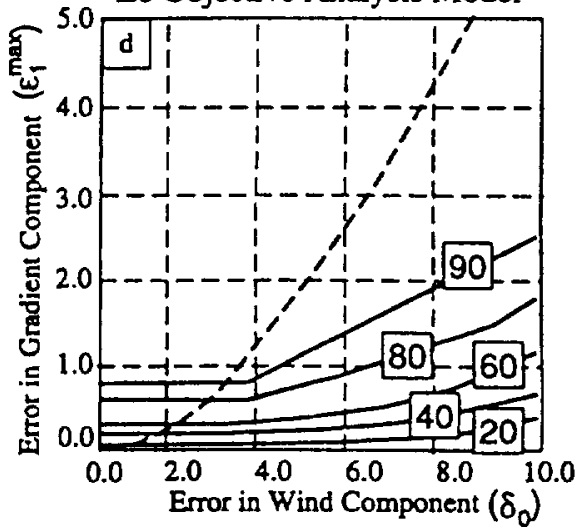

FiG. 8. Error in the wind gradient components as a function of the error in the wind components assuming determined from the observing system simulation experiment (solid lines) and the parameterization (dashed lines) illustrated schematically in Fig. 7. An rms error of $1.5 \mathrm{~m} \mathrm{~s}^{-1}$ in the wind components was assumed. The solid lines denote cumulative percentiles (percentile values indicated in boxes) and indicate the percentage of cases that had error magnitude equal to or less than the value indicated on the ordinate. The ordinate values have units of $10^{-5} \mathrm{~s}^{-1}$ and the abscissa as units of meters per second.

where $\epsilon_{1}^{\max }$ is in units of $10^{-5} \mathrm{~s}^{-1}$ for the wind gradient components and $10^{-5} \mathrm{~K} \mathrm{~m}^{-1}$ for the temperature gradient components. Since, as $\delta_{0}$ becomes greater than $\delta_{0}^{\text {lim }}$, $\epsilon_{1}^{\max }$ increases nearly linearly with increasing $\delta_{0}$, we approximate this increase with a straight line. Therefore, $m$ is the slope of that line and $b$ is the intercept. The constants of the parameterization are listed in Table 6.

Practical application of Eq. (2) requires that the central observations of the temperature and horizontal wind not be included in the objective analysis scheme. However, we consider estimation of uncertainty so critical for quantitative application of the results that withholding the central observation for quality control purposes is necessary. In practice, after an objective analysis model has been applied to the available data, the interpolated value of $u, v$, or $T$ is compared to the central observation of $u, v$, or $T$ to determine the magnitude of $\delta_{0}$. The uncertainty in the spatial derivative, $\epsilon_{1}^{\max }$, then follows from application of Eq. (2). The resulting value of $\epsilon_{1}^{\max }$ can be interpreted as the magnitude of the uncertainty in the spatial derivatives at the $90 \%$ confidence level.

\section{Summary and conclusions}

The proposed use of dynamic and thermodynamic fields that have been derived diagnostically from wind profiler and radiosonde networks by objective analysis has led us to consider the accuracy of those fields. Previous authors have examined this problem from a theoretical aspect but have not addressed the issue of establishing practical estimates of accuracy when considering actual observations in an operational mode. The uncertainty in the diagnosed values produced by $\mathrm{rms}$ observational error alone is a significant fraction of the scale value ( see Tables 1 and 2 ) of each diagnostic term 

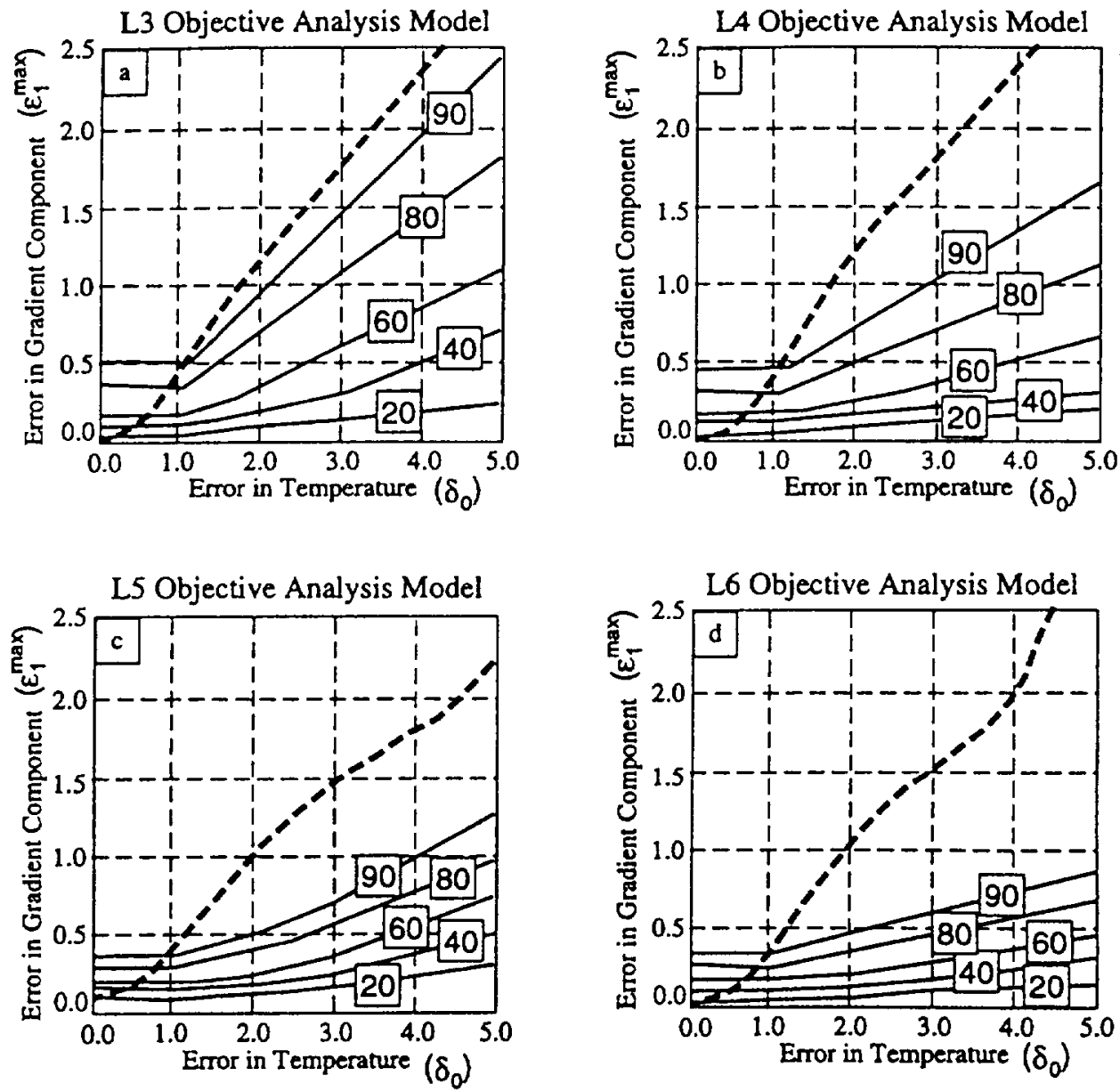

Fig. 9. As in Fig. 8 except for the temperature gradient components as a function of error in temperature. The ordinate has units of $10^{-5} \mathrm{~K} \mathrm{~m}^{-1}$ and the abscissa has units of kelvins. An ms error of $0.5 \mathrm{~K}$ in the temperature was assumed.

regardless of the objective analysis model being used. However, error introduced by the misspecification of the approximating functional model (truncation error) can also produce substantial error. The case study described above shows that the truncation error can seriously bias objective analysis results even in meteorological situations influenced primarily by synopticscale features.

When considering atmospheric data, it is generally impossible to separate the influence of observational and truncation error in the objective analysis results. Given the magnitudes of error that tend to occur in the approximation of horizontal derivatives from data, some idea of the statistical uncertainty in objective analysis results is crucial for further quantitative application of results. The results of the OSSE indicate that in nearly all cases, the quantity under consideration could be estimated to within the scale value of that quantity. However, the results, summarized in the Table 5 also show that well-resolved situations (error less than $25 \%$ of the scale value) occur in no more than one-half of all cases for terms dependent on the diagnosed spatial derivatives.

Scientific use of the objectively analyzed wind and temperature data dictate that the uncertainty in the results be known or at least estimated to reasonable accuracy. Since neither the actual observational error nor the truncation error can be known, we derived a parameterization for the uncertainty in the spatial derivatives $\left(\epsilon_{1}^{\max }\right)$ based on the observed error in the objectively analyzed wind components and temperature $\left(\delta_{0}\right)$. The $\epsilon_{1}^{\max }$ tends to reach a lower limit $\left(\epsilon_{1}^{\mathrm{lim}}\right)$ for some particular value of $\delta_{0}$. This lower limit is the maximum precision that can be prescribed to any diagnosis of a firstorder spatial derivative and depends primarily on the ms error in the observations. The $\epsilon_{t}^{\text {lim }}$ decreases substantially (i.e., the precision increases) as the overdetermination of the least squares solution is increased.

Several additional conclusions can be drawn from the results presented here. 
TABLE 6. Constants of the error parameterization [Eq (2)] discussed in the text. For the wind component gradient terms (ug) the units of $m, b, \epsilon_{0}^{\text {lum }}, \delta_{0}^{\text {him }}$ are $10^{-5} \mathrm{~m}^{-1}, 10^{-5} \mathrm{~s}^{-1}, 10^{-5} \mathrm{~s}^{-1}$, and $\mathrm{m} \mathrm{s}^{-1}$, respectively. For the temperature gradient components $(\mathrm{Tg})$ the units of $\mathrm{m}, b, \epsilon_{0}^{\lim }, \delta_{0}^{\lim }$ are $10^{-5} \mathrm{~m}^{-1}, 10^{-5} \mathrm{~K} \mathrm{~m}^{-1}, 10^{-3} \mathrm{~K} \mathrm{~m}^{-1}$, and $\mathrm{K}$, respectively.

\begin{tabular}{|c|c|c|c|c|c|c|c|c|}
\hline \multirow[b]{2}{*}{ Model } & \multicolumn{4}{|c|}{ ug } & \multicolumn{4}{|c|}{$\mathrm{Tg}$} \\
\hline & $m$ & $b$ & $\epsilon_{0}^{\mathrm{hm}}$ & $\delta_{0}^{\text {lim }}$ & $m$ & $b$ & $\epsilon_{0}^{\lim }$ & $\delta_{0}^{\lim }$ \\
\hline L3 & 0.62 & -0.79 & 1.30 & 3.30 & 0.53 & -0.15 & 0.44 & 0.95 \\
\hline LA & 0.42 & -0.36 & 1.10 & 3.30 & 0.18 & -0.31 & 0.37 & 1.10 \\
\hline L5 & 0.34 & -0.17 & 0.97 & 3.40 & 0.15 & 0.25 & 0.33 & 1.10 \\
\hline L6 & 0.22 & 0.02 & 0.75 & 3.40 & 0.12 & 0.19 & 0.29 & 1.00 \\
\hline
\end{tabular}

1) Hourly objective analysis products derived from wind profiler polygons of less than the six-station inner array and the algorithms considered in this paper should be considered of insufficient accuracy to perform quantitative evaluation of kinematic atmospheric structure.

2) Unless careful visual inspection suggests otherwise, diagnoses of thermal gradients and advective tendencies of temperature using data from the five ARMsponsored radiosonde sites around and within the innerarray region should also be considered of insufficient accuracy to generate boundary conditions for singlecolumn modeling applications.

3) Despite the previous two points, closely monitoring the experimental data stream using, initially, the uncertainty parameterization presented earlier and then careful scientific evaluation, should allow identification of cases that are sufficiently well characterized. It should be realized, however, that these cases will be limited in number and will likely exclude many typical synoptic-scale dynamical regimes, such as jet streak entrance or exit regions and frontal zones.

In the context of the single-column modeling application, our results and conclusions are simultaneously encouraging and discouraging. The encouraging aspect is that there are a wide variety of situations in which the diagnosed fields are of sufficient accuracy to permit their use as forcing functions for SCMs. Furthermore, we have suggested a simple, and somewhat crude, parameterization that can be used to decide when the diagnosed fields can be used. The discouraging aspect is that the fields are most likely to be inaccurate in situations where the actual fields have strong spatial and temporal gradients. These often will be the very conditions in which one would like to use the diagnosed fields. An alternative to using the objectively analyzed fields is to use fields produced by data assimilation models. In some sense, data assimilation model fields may be seen as objectively analyzed or interpolated by the model itself, but in a way that produces balanced and consistent results. Considerable research remains to be done using both objectively analyzed fields and data assimilation output in order to evaluate the usefulness and accuracy of these two approaches when applied in an operational mode.

Acknowledgments. The useful and insightful comments of Dr. Bruce Albrecht and Dr. Eugene Clothiaux are greatly appreciated. We would also like to thank Dr. Fred Sanders and several anonymous reviewers for providing extensive and helpful reviews of earlier drafts of this paper. Support for this research was funded in part by the Environmental Sciences Division of U.S. Department of Energy (under Grant DE-FG0290ER61071) and NASA Research Grant NAG-1-1095.

\section{REFERENCES}

Akima, H., 1978: A method of bivariate interpolation and smooth surface fitting for values given at irregularly distributed points. ACM Trans. Math. Software, 4, 148-159.

- 1984: On estimating partial derivatives for bivariate interpolation of scattered data. Rocky Mt. J. Math., 14, 41-52.

Bellamy, J. C., 1949: Objective calculations of divergence, verical velocity and vorticity. Bull. Amer. Meteor. Soc., 30, 45-49.

Belt. C. L., and H. E. Fuelberg. 1982: Effects of random errors in rawinsonde data on derived kinematic quantities. Mon. Wea. Rev., 110, 91-101.

Benjamin, S. G., 1989: An isentropic meso-alpha scale analysis system and its sensitivity to aircraft and surface observations. Mon. Wea. Rev., 117, 1586-1605.

Davies-Jones, R., 1993: Useful formulas for computing divergence, vorticity, and their errors from three or more stations. Mon. Wea. Rev., 121, 713-725.

Doswell, C. A., III, and F. Caracena, 1988: Derivative estimation from marginaliy sampled vector point functions. J. Atmos. Sci., $45,242-253$.

Endlich, R. M., and J. R. Clark, 1963: Objective computation of some meteorological quantities. J. Appl. Meteor., 2, 66-81.

Kaylor, R. E., 1977: Filtering and decimation of digital time series. Tech. Note BN850, Inst. Phys. Sci. Tech., University of Maryland. College Park, MD, 42 pp.

Mace, G. G., 1994: Development of large-scale diagnossic analysis techniques applicable to regional arrays of wind profilers and radiosondes. Ph.D. dissertation, Pennsylvania State University, 262 pp.

, D. O'C. Starr, T. P. Ackerman, and P. Minnis, 1995: Examination of coupling between an upper-tropospheric cloud system and synoptic-scale dynamics diagnosed from wind profiler and radiosonde data. J. Armos. Sci., 52, 4094-4127.

Michaels, P., 1994: Estimating advective tendencies from field measurements. Mon. Wea. Rev., 122, 2202-2209.

NASA, 1991: FIRE Cirrus Intensive Field Observations-II: Operations Plan.

Stokes, G. M., and S. E. Schwartz, 1994: The Atmospheric Radiation Measurement (ARM) Program: Programmatic background and design of the cloud and radiation test bed. Bull. Amer. Meteor. Soc., 75, $1201-1221$.

Strauch, R. G., B. L. Weber, A. S. Frisch, C. G. Little. D. A. Merrith, K. P. Moran, and D. C. Welsh, 1987: The precision and relative accuracy of profiler wind measurements. J. Atmos. Oceanic. Technol., 4, 563-571.

Thiebaux, H. J., and M. A. Pedder, 1987: Sparial Objective Analysis, with Applicarions in Amospheric Science. Academic Press Inc., 299 pp.

Zamora, R. J.. M. A. Shapiro, and C. A. Doswell III. 1987: The diagnosis of upper-topospheric divergence and ageostrophic wind using profiler wind observations. Mon. Wea. Rev., 115, 871-884.

- B. L. Weber, and D. C. Welsh, 1994: The accuracy of divergence estimates calculated using the linear vector point function method and three profilers. Mon. Wea. Rev., 122, 2603-2606. 